DOI: 10.12957/demetra.2017.26643

\title{
Fatores associados ao aleitamento materno exclusivo em crianças menores de seis meses em Guarapuava-PR
}

\section{Factors associated with exclusive breastfeeding in children under the age of six months in Guarapuava-PR}

\author{
Larissa Naiana Rauber' \\ Thais Fernanda Sehnen de Souza \\ Priscilla Negrão de Moura' \\ Catiuscie Cabreira da Silva' \\ Luana Bernardi' \\ Paula Chuproski Saldan \\ 'Universidade Estadual do Centro-Oeste, \\ Departamento de Nutrição. Guarapuava-PR, Brasil. \\ Correspondência / Correspondence \\ Paula Chuproski Saldan \\ Departamento de Nutrição Campus CEDETEG \\ Rua Simeão Camargo Varela de Sá, 03 Vila Carli \\ - CEP 85040-080 Guarapuava, PR, Brasil \\ E-mail: pchuproski@unicentro.br
}

\section{Resumo}

Objetivo: Analisar os fatores associados ao Aleitamento Materno Exclusivo (AME) em crianças menores de seis meses de vida em Guarapuava-PR. Metodologia: Foram analisados dados das crianças menores de seis meses de vida que participaram da Campanha Nacional de Vacinação contra Poliomielite 2012, em Guarapuava-PR. A coleta ocorreu por aplicação de questionário contendo perguntas referentes à alimentação das crianças no dia anterior à pesquisa, características das crianças e das mães. Para a identificação dos fatores associados ao AME, foram construídos modelos de regressão de Poisson, obtendo-se Razões de Prevalências (RP) em modelos bivariados e múltiplo. Consideraram-se fatores associados ao AME as variáveis que mantiveram $\mathrm{p}<0,05$ na análise múltipla. Resultados: Foram estudadas informações de 459 crianças. Os fatores independentes relacionados à interrupção do AME em menores de seis meses de vida foram o uso de bicos (RP 1,79; IC $1,57-2,05)$ e não ser amamentado na primeira hora de vida (RP 1,19; IC 1,05-1,34). Conclusões: É importante avaliar os fatores que influenciam a amamentação a fim de propor estratégias mais efetivas para proteção desta prática, em prol do aumento da duração do Aleitamento Materno Exclusivo.

Palavras-chave: Aleitamento Materno. Lactente. Estudos Transversais.

\section{Abstract}

Objective: To analyze the factors associated with exclusive breastfeeding in children under the age of six months in 
Guarapuava-PR, Brazil. Methods: An analysis was made of data from children under the age of six months who participated in The National Vaccination Campaign against Poliomyelitis 2012, in Guarapuava-PR, Brazil. The data were collected by means of a questionnaire with questions about feeding children the day before the survey, characteristics of children and mothers. To identify factors associated with exclusive breastfeeding, Poisson regression models were constructed, obtaining Prevalence Ratios (PR) in bi-variate and multiple models. The only factors considered to be associated with breastfeeding were those with $\mathrm{p}<0.05$ in the multiple models. Results: Data from 459 children were analyzed. The independent factors associated with the interruption of exclusive breastfeeding in this study were the use of artificial nipples (PR 1.79; CI 1.57-2.05) and not being breastfed in the first hour of life (PR 1.18; CI 1.05-1.34). Conclusions: It is important to evaluate the factors that influence breastfeeding in order to offer more effective strategies for the protection of this action, aiming to increase the duration of exclusive breastfeeding.

Keywords: Breast Feeding. Infant. Cross-Sectional Studies.

\section{Introdução}

O leite materno (LM) é o único alimento completo que contém os nutrientes necessários para alimentar o bebê nos primeiros seis meses de vida. ${ }^{1}$ Desde 2001, em razão das evidências dos benefícios do leite humano, a Organização Mundial da Saúde (OMS) recomenda que a criança seja amamentada de forma exclusiva nos primeiros seis meses de vida e que somente a partir desse período seja iniciada a alimentação complementar (AC), com alimentos apropriados e seguros, com continuidade do aleitamento materno (AM) por dois anos ou mais de vida. ${ }^{2}$

$\mathrm{O}$ aleitamento materno exclusivo (AME) nos seis primeiros meses de vida constitui uma intervenção fundamental para a sobrevivência infantil e confere proteção contra infecções respiratórias e diarreia. ${ }^{3,4}$ Crianças não amamentadas apresentam maior chance de morrer por doenças infecciosas nos primeiros dois meses de vida que aquelas amamentadas. ${ }^{5}$ Foi estimado que as mortes de 823 mil crianças e 20 mil mães poderiam ser evitadas anualmente com a ampliação mundial da amamentação, ainda com benefício adicional de economia de 302 bilhões de dólares. ${ }^{6,7}$

Além disso, o AM diminui o risco de alergias e de doenças crônicas não transmissíveis, possui efeito positivo na inteligência, melhora o desenvolvimento da cavidade bucal, tem menor custo financeiro para a família e promove o vínculo da mãe com o filho. ${ }^{1}$ 
A amamentação também traz benefícios para a mãe, estima-se que para cada ano de amamentação haja uma diminuição de 4,3\% do risco de câncer de mama e de $15 \%$ do de diabetes, e que para cada mês de amamentação o risco de câncer de ovário seja $2 \%$ menor. ${ }^{8,9}$ Ainda, favorece a perda de peso no pós-parto e prolonga a amenorreia lactacional. ${ }^{10}$

A prevalência de AME no Brasil, aos dois e três meses de vida, aumentou de 26,4\%, em 1996, para 48,3\%, em 2006, ${ }^{11}$ evidenciando que, apesar do aumento na prevalência, esses valores ainda estão aquém das recomendações. Dados da II Pesquisa de Prevalência de Aleitamento Materno nas Capitais Brasileiras e Distrito Federal de 2008 indicaram prevalências de AME de 41\% em menores de seis meses de vida e duração mediana do AME de 54,1 dias, inferior ao recomendado. ${ }^{12}$

No Brasil, estudos demonstram que fatores como escolaridade, idade materna, práticas associadas ao parto e ao nascimento têm influência sobre o AM. ${ }^{13,14}$ Outros estudos nacionais advertem que o período de introdução alimentar é precoce, a consistência e os alimentos oferecidos são inadequados e fatores como primiparidade, idade, escolaridade, trabalho e renda materna influenciam a alimentação da criança. ${ }^{15-17}$

A prevalência de AME em menores de seis meses de vida em Guarapuava, segundo dois estudos locais, é baixa. Em 2004, a prevalência de AME foi de 37,3\% e em 2012, de 36,0\%. ${ }^{18,19}$ No município, a duração mediana do AME entre menores de seis meses de vida, encontrada em 2012, foi de 48,2 dias. ${ }^{19}$ De tal modo, o objetivo deste estudo foi analisar os fatores associados ao AME em crianças menores de seis meses de vida no município de Guarapuava-PR, a fim de propor estratégias mais efetivas para o enfrentamento da baixa prevalência dessa prática.

\section{Metodologia}

Para a realização deste estudo, foi utilizada parte dos dados coletados em um estudo transversal conduzido durante a Campanha Nacional de Vacinação contra Poliomielite 2012, em GuarapuavaPR. ${ }^{19}$ A população de estudo foi o conjunto de crianças menores de dois anos de vida que compareceram aos postos de vacinação da área urbana e rural do município durante a campanha, porém para este estudo fez-se uso dos dados das crianças menores de seis meses de vida.

O tamanho da amostra foi estimado com base na prevalência de AME em menores de seis meses de vida, com parâmetro de $40 \%$ entre 2-3 meses, segundo estudo local, ${ }^{20}$ e erro amostral de $9 \%$. As estimativas foram obtidas aplicando a expressão algébrica de Lwanga \& Lemeshow ${ }^{21}$ descrita abaixo:

$$
n_{1}=\frac{P \cdot Q}{(d / 1,96)^{2}}
$$


Posteriormente, foi aplicado ajuste de não resposta de $5 \%$ e efeito de delineamento de 1,4. O cálculo amostral final de crianças menores de seis meses de vida foi de 550, conforme pode ser visto no quadro 1.

Quadro 1. Cálculo da amostra do estudo

\begin{tabular}{|c|c|c|c|c|c|c|c|c|c|}
\hline Grupo & Estimador & Parâmetro & $\begin{array}{c}\text { Erro } \\
\text { amostral }\end{array}$ & $\begin{array}{c}\text { Nível de } \\
\text { confiança }\end{array}$ & $\begin{array}{c}\mathrm{n} \text { da } \\
\text { faixa }\end{array}$ & $\begin{array}{c}\mathrm{n} \\
\text { estendido }\end{array}$ & $\begin{array}{c}\text { deff } \\
1,4\end{array}$ & $\begin{array}{c}\text { Não } \\
\text { resposta } \\
(5 \%)\end{array}$ & $\begin{array}{c}\text { n final } \\
\text { (para } \\
\text { análise } \\
\text { múltipla) }\end{array}$ \\
\hline $\begin{array}{c}<6 \\
\text { meses }\end{array}$ & $\begin{array}{c}\text { AME (2 a } \\
3 \text { meses } \\
\text { completos) }\end{array}$ & $40 \%$ & $9 \%$ & $95 \%$ & $\begin{array}{c}(2-3 \\
\mathrm{m}) \\
114\end{array}$ & $\begin{array}{c}(<6 \\
\text { meses }) \\
342\end{array}$ & 478,8 & 502,7 & 550 \\
\hline
\end{tabular}

AME=Aleitamento Materno Exclusivo, deff=sigla em inglês "design effect" - efeito do delineamento do estudo.

O estudo adotou amostragem por conglomerados, e considerando que as crianças não estavam distribuídas uniformemente nos vários postos de vacinação (conglomerados), foi aplicado sorteio em dois estágios, ${ }^{22}$ com probabilidade proporcional ao tamanho dos conglomerados. No primeiro estágio, foram sorteados os postos de vacinação e no segundo, de forma sistemática, foram sorteadas as crianças na fila de vacinação em cada posto. Foram sorteados 32 postos de vacinação.

A coleta dos dados foi realizada no período de 11 a 29 de junho de 2012. O instrumento de coleta foi um questionário baseado e modificado a partir daquele aplicado em campanhas de vacinação do projeto Amamentação e Municípios, do Instituto de Saúde da Secretaria de Saúde de São Paulo, ${ }^{13}$ adotado pelo Ministério da Saúde para realização da II Pesquisa de Prevalência de AM nas Capitais Brasileiras e Distrito Federal, em 2008. ${ }^{12}$ As questões sobre alimentação foram baseadas em todos os prováveis alimentos que a criança ingeriu no dia anterior ao da entrevista (LM, água, chá, outros leites, mingau, suco de fruta, frutas, comida de sal - comida de panela, papa ou sopa) (Recordatório de 24 horas), e as demais questões eram relativas à criança, mãe e serviço de saúde.

Para a coleta dos dados, foram selecionados 118 estudantes voluntários dos cursos de Nutrição e Enfermagem de universidade local que receberam treinamento de 4 horas. Para a identificação dos fatores associados ao AME, foram construídos modelos de regressão de Poisson, obtendo-se Razões de Prevalências (RP) em modelos bivariados e múltiplo, estimadas por pontos e Intervalos de Confiança de 95\% (IC 95\%). A variável dependente deste estudo foi o indicador AME em menores 
de seis meses de vida (sim ou não). As variáveis independentes foram: sexo da criança (feminino ou masculino), tipo de parto (vaginal ou cesárea), peso ao nascer ( $\geq 2500$ ou $<2500$ gramas), amamentação na primeira hora de vida (sim ou não), uso de bicos - mamadeira, chuca e chupeta - (sim ou não), serviço de saúde onde a criança se consulta (rede pública ou privada/convênio), paridade materna (primípara ou multípara), idade materna ( $\geq 20$ ou $<20$ anos), escolaridade materna ( $\geq 8$ ou $<8$ anos de estudo), trabalho materno (trabalha fora, não trabalha fora ou licença maternidade), residir com o pai da criança (sim ou não) e área de residência (urbana ou rural). A idade da criança em meses (0-1 mês, 1-2, 2-3, 3-4, 4-5 meses) foi considerada como variável de controle. As categorias de referência foram codificadas em 0 e as de risco, em 1.

Modelos bivariados foram construídos contendo cada uma das variáveis independentes e a variável dependente. As variáveis para as quais se obtiveram valores de $\mathrm{p} \leq 0,20$ (teste de Wald) foram selecionadas para o modelo múltiplo. Para elaboração do modelo final, foi utilizado o procedimento stepwise forward, permanecendo no modelo as variáveis que apresentaram $\mathrm{p}<0,05$.

As estimativas foram calculadas levando em consideração o efeito do desenho (módulo survey). A análise dos dados foi processada no programa Stata versão 12.0 (StataCorp, College Station, Texas, EUA).

O presente estudo foi aprovado pelo Comitê de Ética em Pesquisa da Escola de Enfermagem de Ribeirão Preto da Universidade de São Paulo (Ofício CEP-EERP/USP - 253/2012).

\section{Resultados}

Neste estudo, foram analisados os fatores associados à prática de AME de 459 crianças menores de seis meses de vida. O número de recusas do estudo original foi de 149 (8,06\%), no qual foram coletados dados de 1.848 crianças menores de dois anos de vida.

Na tabela 1 estão descritas as principais características das mães e crianças participantes do estudo. Observou-se que $51,63 \%$ das crianças nasceram de parto vaginal, $93,90 \%$ com peso adequado ao nascer e 76,69\% foram amamentadas na primeira hora de vida. Em relação às mães, verificou-se que $67,54 \%$ tinham vinte ou mais anos de idade e $66,45 \%$, oito ou mais anos de estudo. 
Tabela 1. Características das mães e crianças menores de seis meses de idade participantes do estudo em Guarapuava - PR, 2012.

\begin{tabular}{|c|c|c|}
\hline Características & $\mathbf{N}$ & $\%$ \\
\hline \multicolumn{3}{|l|}{ Faixa etária (meses) } \\
\hline $0 \vdash-1$ & 75 & 16,34 \\
\hline $1 \vdash-2$ & 78 & 16,99 \\
\hline $2 \vdash-3$ & 76 & 16,56 \\
\hline $3 \vdash-4$ & 80 & 17,43 \\
\hline $4 \vdash-5$ & 74 & 16,12 \\
\hline $5 \vdash-6$ & 76 & 16,56 \\
\hline \multicolumn{3}{|l|}{ Sexo } \\
\hline Feminino & 231 & 50,33 \\
\hline Masculino & 228 & 49,67 \\
\hline \multicolumn{3}{|l|}{ Tipo de parto } \\
\hline Vaginal & 237 & 51,63 \\
\hline Cesárea & 221 & 48,15 \\
\hline Não informado* & 1 & 0,22 \\
\hline \multicolumn{3}{|l|}{ Peso ao nascer (gramas) } \\
\hline$\geq 2.500$ & 431 & 93,90 \\
\hline$<2.500$ & 26 & 5,66 \\
\hline Não informado* & 2 & 0,44 \\
\hline \multicolumn{3}{|l|}{ Mamou primeira hora de vida } \\
\hline Sim & 352 & 76,69 \\
\hline Não & 99 & 21,57 \\
\hline Não informado* & 8 & 1,74 \\
\hline \multicolumn{3}{|l|}{ Uso de bicos } \\
\hline Sim & 165 & 35,95 \\
\hline Não & 289 & 62,96 \\
\hline Não informado* & 5 & 1,09 \\
\hline
\end{tabular}




\begin{tabular}{|c|c|c|}
\hline Características & $\mathbf{N}$ & $\%$ \\
\hline \multicolumn{3}{|l|}{ Serviço de saúde } \\
\hline Rede pública & 310 & 67,54 \\
\hline Rede privada/convênio & 136 & 29,63 \\
\hline Não informado* & 13 & 2,83 \\
\hline \multicolumn{3}{|l|}{ Paridade materna** } \\
\hline Primípara & 230 & 50,11 \\
\hline Multípara & 196 & 42,70 \\
\hline Não informado* & 33 & 7,19 \\
\hline \multicolumn{3}{|l|}{ Idade materna (anos)** } \\
\hline$<20$ & 11 & 24,62 \\
\hline$\geq 20$ & 310 & 67,54 \\
\hline Não informado* & 36 & 7,84 \\
\hline \multicolumn{3}{|l|}{ Escolaridade materna (anos)** } \\
\hline$\geq 8$ & 305 & 66,45 \\
\hline$<8$ & 121 & 26,36 \\
\hline Não informado* & 33 & 7,19 \\
\hline \multicolumn{3}{|l|}{ Trabalho materno** } \\
\hline Trabalha fora & 67 & 14,60 \\
\hline Não trabalha fora & 298 & 64,92 \\
\hline Licença maternidade & 60 & 13,07 \\
\hline Não informado* & 34 & 7,41 \\
\hline \multicolumn{3}{|l|}{ Reside com o pai da criança** } \\
\hline Sim & 367 & 79,96 \\
\hline Não & 59 & 12,85 \\
\hline Não informado* & 33 & 7,19 \\
\hline \multicolumn{3}{|l|}{ Área de residência } \\
\hline Urbana & 411 & 89,54 \\
\hline Rural & 48 & 10,46 \\
\hline
\end{tabular}

*Dados não respondidos pelo acompanhante da criança.

**Dados coletados quando o acompanhante da criança era a mãe. 
Na análise bivariada (Tabela 2), observou-se associação significativa entre o AME e as seguintes variáveis: tipo de parto $(\mathrm{p}=0,186)$, peso ao nascer $(\mathrm{p}=0,082)$, amamentação na primeira hora de vida $(\mathrm{p}=0,001)$, uso de bicos $(\mathrm{p}<0,001)$, paridade materna $(\mathrm{p}=0,022)$, escolaridade materna $(\mathrm{p}=0,182)$, trabalho materno $(\mathrm{p}=0,003)$ e área de residência $(\mathrm{p}=0,208)$.

Tabela 2. Modelos bivariados considerando o aleitamento materno exclusivo em menores de seis meses e variáveis independentes em Guarapuava - PR, 2012.

\begin{tabular}{|c|c|c|c|}
\hline Variável & RP bruta & IC $95 \%$ & p valor* \\
\hline \multicolumn{4}{|l|}{ Sexo da criança } \\
\hline Feminino & 1,00 & & \\
\hline Masculino & 0,95 & $(0,78-1,15)$ & 0,593 \\
\hline \multicolumn{4}{|l|}{ Tipo de parto } \\
\hline Vaginal & 1,00 & & \\
\hline Cesárea & 0,91 & $(0,79-1,05)$ & $0,186^{* *}$ \\
\hline \multicolumn{4}{|l|}{ Peso ao nascer (gramas) } \\
\hline$\geq 2500$ & 1,00 & & \\
\hline$<2500$ & 1,22 & $(0,97-1,53)$ & $0,082 * *$ \\
\hline \multicolumn{4}{|l|}{ Mamou primeira hora vida } \\
\hline Sim & 1,00 & & \\
\hline Não & 1,28 & $(1,12-1,46)$ & $0,001 * *$ \\
\hline \multicolumn{4}{|l|}{ Uso de bicos } \\
\hline Não & 1,00 & & \\
\hline Sim & 1,93 & $(1,68-2,23)$ & $<0,001 * *$ \\
\hline \multicolumn{4}{|l|}{ Serviço de saúde } \\
\hline Rede pública & 1,00 & & \\
\hline Rede privada/convênio & 0,93 & $(0,79-1,09)$ & 0,353 \\
\hline \multicolumn{4}{|l|}{ Paridade materna } \\
\hline Multípara & 1,00 & & \\
\hline Primípara & 1,23 & $(1,03-1,47)$ & $0,022 * *$ \\
\hline
\end{tabular}


Continuação da Tabela 2

\begin{tabular}{lccc}
\hline \multicolumn{1}{c}{ Variável } & RP bruta & IC 95\% & p valor* \\
\hline $\begin{array}{l}\text { Idade materna (anos) } \\
\geq 20\end{array}$ & 1,00 & & \\
$<20$ & 1,03 & $(0,84-1,25)$ & 0,775 \\
Escolaridade materna (anos) & & & \\
$\geq 8$ & 1,00 & & $\mathbf{0 , 1 8 2 * *}$ \\
$<8$ & 1,10 & $(0,95-1,27)$ & \\
Trabalho materno & & & $\mathbf{0 , 0 0 2 * *}$ \\
$\quad$ Trabalha fora & 1,00 & & $\mathbf{0 , 0 0 3} * *$ \\
$\quad$ Não trabalha fora & 0,76 & $(0,64-0,90)$ & \\
$\quad$ Licença maternidade & 0,63 & $(0,48-0,84)$ & 0,662 \\
Reside com o pai da criança & & & \\
$\quad$ Não & 1,00 & & $0,208^{* *}$ \\
Sim & 0,96 & $(0,78-1,17)$ & \\
Area de residência & & & \\
Urbana & 1,00 & $(0,95-1,27)$ & \\
Rural & 1,10 & & \\
\hline
\end{tabular}

$\mathrm{RP}=$ Razão de Prevalência, IC 95\%= Intervalo de Confiança, *Teste de Wald,**variáveis selecionadas para o modelo múltiplo.

Os fatores independentes associados à interrupção do AME em crianças menores de seis meses de vida em Guarapuava-PR foram: fazer uso de bicos $(\mathrm{RP}=1,79$; $\mathrm{IC}=1,57-2,05)$ e não ser amamentado na primeira hora de vida $(\mathrm{RP}=1,19 ; \mathrm{IC}=1,05-1,34)$, após controlado o efeito do peso ao nascer da criança, tipo de parto, paridade materna, escolaridade, idade materna, trabalho materno, área de residência e idade da criança em meses (Tabela 3). 
Tabela 3. Fatores associados à interrupção do aleitamento materno exclusivo em crianças menores de seis meses de idade em Guarapuava - PR, 2012.

\begin{tabular}{lll}
\hline \multicolumn{1}{c}{ Variável } & RP bruta (IC 95\%) & \multicolumn{1}{c}{ RP ajustada (IC95\%)* } \\
\hline $\begin{array}{ll}\text { Uso de bicos } \\
\text { Não }\end{array}$ & 1,00 & 1,00 \\
$\quad$ Sim & $1,93(1,68-2,23)$ & $1,79(1,57-2,05)$ \\
Mamou primeira hora de vida & & \\
Sim & 1,00 & 1,00 \\
Não & $1,28(1,12-1,46)$ & $1,19(1,05-1,34)$ \\
\hline
\end{tabular}

Variáveis controladas para o peso ao nascer da criança, tipo de parto, paridade materna, escolaridade, idade materna, trabalho materno, área de residência e idade da criança em meses.

RP= Razão de Prevalência, IC 95\%= Intervalo de Confiança,*Regressão de Poisson.

\section{Discussão}

Este estudo possibilitou analisar as características das mães e crianças participantes e avaliar os fatores independentes associados à interrupção do AME entre crianças menores de seis meses de vida em Guarapuava-PR, que foram: fazer uso de bicos e não ser amamentado na primeira hora de vida.

A associação do abandono do aleitamento e do uso de bicos (mamadeira e chupeta) é bem reportada na literatura. Pesquisa com crianças menores de seis meses de vida em Campanha de Multivacinação em Uberlândia-MG, em 2008, referiu o uso de chupetas e bicos $(\mathrm{OR}=4,2 ; \mathrm{IC}=2,8$ $6,3)$ associado com o desmame precoce, ${ }^{23}$ semelhante ao presente estudo. Pellegrinelli et al., ${ }^{24} \mathrm{em}$ investigação com 9.474 mães, entre os anos 2009 e 2011, analisou informações sociodemográficas, antecedentes obstétricos, dados sobre aleitamento, mamadeira e chupeta, e também encontrou o uso de mamadeira associado à menor prevalência de AME.

Estudo transversal sobre a situação da amamentação e da AC em município do Vale do Ribeira, São Paulo, revelou que as crianças que não usavam chupeta ( $\mathrm{RP}=1,95$; IC 95\%=1,15-3,30) foram as que tiveram maior possibilidade de AME. ${ }^{25}$ Campagnolo et al. ${ }^{26} \mathrm{em}$ pesquisa realizada em Porto Alegre-RS, no ano de 2008, com 1.099 crianças menores de um ano de vida, também encontraram maior frequência de AME entre crianças que não usavam chupeta.

Ducci et al., ${ }^{27}$ em estudo transversal em Rolândia-PR, concluíram que crianças menores de quatro meses de vida que não utilizavam mamadeira apresentaram prevalência de AME 13,56 vezes maior à das crianças que a utilizavam $(\mathrm{p}<0,001)$. 
O desmame precoce causado pelo uso da mamadeira pode ser explicado por uma confusão gerada pela exposição a diferentes formas de sucção, as crianças, após experimentar a mamadeira, passam a apresentar dificuldade quando vão mamar ao peito. ${ }^{1}$

Muitas mães ofertam chupetas, com frequência, como um mecanismo para diminuir e espaçar as mamadas, para suprir as necessidades de sucção quando ocorre a volta ao trabalho, a fim de acalmar os bebês e auxiliá-los a dormir. ${ }^{28,29}$ Dadalto et al. ${ }^{30}$ investigando inicialmente 62 mães de recém-nascidos pré-termo em Vitória-ES, observaram que 34,6\% das mães ofereciam chupetas para acalmar a criança; 26,9\%, para ajudar a adormecer mais rápido; 23,1\%, para aumentar o tempo de sucção por estar mamando a noite toda; 11,5\%, porque o bebê iniciou sução digital; e 3,8\%, para ver se ele precisava da chupeta. Esse estudou também evidenciou que a idade para início do hábito de chupeta em 26,9\% ocorreu anterior aos três meses da criança, e em 73,1\%, na idade de três meses ou mais.

Estudo de coorte iniciado em 2000, envolvendo 250 crianças nascidas no Hospital Amigo da Criança, em Porto Alegre, verificou a prática do uso de chupeta com obtenção de dados na maternidade, no final do primeiro mês, no segundo, quarto e sexto mês de vida. Encontrou-se que 20,4\% das mães levaram chupeta para a maternidade e 1,2\% ofereceu ao recém-nascido. No primeiro mês, $61,6 \%$ das crianças localizadas faziam uso de bicos, e dessas, $34,2 \%$ usavam desde a primeira semana de vida. ${ }^{31} \mathrm{Um}$ aspecto cultural regional também está relacionado com o uso de mamadeira e ao abandono do AME. Saldiva et al. ${ }^{32}$ analisaram dados de 18.929 crianças da II Pesquisa de Prevalência de Aleitamento Materno nas Capitais Brasileiras, de 2008, encontrando maior razão de prevalência para consumo de chá nas capitais do Sul entre menores de seis meses de vida. O hábito da introdução de chá pode ser explicado pelo clima frio, além de ser muito utilizado para fins terapêuticos.

Estudo transversal que analisou dados de 34.366 crianças também obtidos da II Pesquisa de Prevalência de Aleitamento Materno nas Capitais e DF, em 2008, avaliando os fatores associados ao uso de chupeta e/ou mamadeira em crianças menores de um ano de vida, encontrou que ter mamado na primeira hora de vida favoreceu a menor frequência de uso de bicos artificiais. ${ }^{29}$

Neves et al., ${ }^{33}$ em pesquisa com 9.060 crianças menores de seis meses de vida residentes da Amazônia Legal e do Nordeste do Brasil, identificaram maior probabilidade de AME entre mães que amamentaram na primeira hora de vida da criança, assim como no presente estudo. Pesquisa longitudinal realizada com 246 mulheres, em 2003, com acompanhamento até o primeiro ano da criança ou até o desmame, encontrou que a primeira mamada após seis horas de vida se associou à menor duração do $\mathrm{AM}(\mathrm{RR}=1,95 \mathrm{IC}=1,03-3,67) \cdot{ }^{34}$

Ramos et al. ${ }^{35}$ realizaram diagnóstico da situação do AM pesquisando 1.963 crianças menores de um ano de vida em 45 municípios do Piauí, no ano de 2006. Entre os resultados, amamentar 
na primeira hora de vida se mostrou associado com a prática da amamentação $(\mathrm{p}<0,001)$. No estudo citado acima, encontrou-se maior significância (82\%) de aleitamento entre as crianças que foram aleitadas no primeiro dia de vida em relação às que não foram (68\%).

Estudo realizado em Feira de Santana-BA com 2.319 crianças menores de um ano de vida, no ano de 2001, encontrou maiores chances de aleitamento exclusivo entre crianças que foram amamentadas no primeiro dia de vida $(40,4 \%)$ e que não utilizavam chupetas $(49,4 \%)$, assim como os dados da presente pesquisa. ${ }^{36}$

A promoção do aleitamento na primeira hora de vida, além de ser fundamental para o sucesso da amamentação exclusiva e continuada, pode ser um fator de proteção contra taxas de mortalidade neonatal, sendo importante a realização desse procedimento ainda na sala de parto. ${ }^{37}$

Will et al. ${ }^{38}$ investigaram os fatores que influenciaram o aleitamento na primeira hora de vida entre 169 crianças de Vitória-ES, no ano de 2009, cadastradas em Unidades de Saúde da Família. Encontraram associação entre amamentar na primeira hora de vida e as variáveis parto vaginal e estar em alojamento conjunto. Estudo transversal analisou dados do município do Rio de Janeiro entre 1999 e 2001, investigando 8.397 mães. Constatou que o AM na primeira hora de vida foi menos prevalente entre os recém-nascidos, com intercorrências após o parto, entre as mães que não tiveram contato com os bebês na sala de parto, entre as que tiveram parto cesárea e cujo parto ocorreu em maternidade privada ou conveniada com o Sistema Único de Saúde. Neste estudo, o parto cesariano reduziu pela metade a prevalência da amamentação na primeira hora nas maternidades locais. ${ }^{39} \mathrm{~A}$ região Sul possui um dos maiores percentuais de parto cesáreo do Brasil, correspondendo a $51,6 \%$ dos partos, sendo fator de risco para a não amamentação na primeira hora de vida. ${ }^{11}$

Como estratégia de proteção e promoção do AM há os Hospitais Amigos da Criança. A amamentação na primeira hora de vida corresponde ao passo 4 da Iniciativa Hospital Amigo da Criança (IHAC), a fim de apoiar a interação mãe-bebê logo após o parto. Esta Iniciativa foi difundida em 1991 com o objetivo de mudar as rotinas hospitalares segundo o cumprimento dos "Dez Passos Para o Sucesso da Amamentação", pela OMS e pelo Fundo das Nações Unidas para a Infância (UNICEF), e não aceitar doações de substitutos do LM. ${ }^{40}$

Apesar de os fatores associados à interrupção do AME em menores de seis meses de vida encontrados neste estudo já serem reconhecidos na literatura, para o município e para o gestor de saúde essas informações são de extrema relevância para o planejamento de ações que visem promover o AM. Os profissionais de saúde que trabalham com obstetrícia precisam promover a amamentação na primeira hora de vida das crianças, dados os benefícios dessa prática. Já o fator uso de bicos é mais difícil de ser mudado e requer esforço de profissionais que trabalham com a amamentação, mas também dos pais e familiares que necessitam repensar seu uso e o que isso implica para a saúde das crianças. 
Como ação mais concreta para melhorar a prevalência de AM no munícipio de Guarapuava, podemos citar a IHAC, e os dois hospitais do município estão tentando o credenciamento desta estratégia. Além dessa, também podemos citar a Estratégia Amamenta e Alimenta Brasil, que visa qualificar os profissionais da atenção básica na temática do AM e AC.

Algumas limitações do estudo consistem na forma de avaliar o AME pelo método do Recordatório de 24 horas, o que pode superestimar essa prática, pois as crianças podem não ter recebido outros líquidos ou alimentos no dia anterior ao da entrevista, porém podem ter recebido em outros dias não avaliados neste estudo. Além disso, alguns dados de crianças ou mães não informados também podem comprometer os resultados do presente estudo, em especial quando as crianças não estavam acompanhadas de suas mães.

\section{Conclusões}

Este estudo possibilitou analisar os fatores associados ao AME em crianças menores de seis meses de vida no município de Guarapuava-PR. Observou-se que fazer uso de bicos e não ser amamentado na primeira hora de vida estão associados à interrupção do AME.

É importante avaliar os fatores que influenciam a amamentação em cada região/município a fim de propor estratégias mais efetivas para proteção da prática do AME, em prol da saúde das crianças. Sendo assim, destaca-se a promoção de ações contínuas direcionadas aos fatores que influenciam o desmame precoce no município, contribuindo para o aumento da duração dessa prática.

\section{Colaboradores}

Rauber LN, de Souza TFS e Saldan PC trabalharam em todas as etapas, desde a concepção do estudo até a revisão da versão final do artigo. Moura PN, da Silva C e Bernardi L participaram da redação do artigo e da sua versão final.

Conflito de Interesses: Os autores declaram não haver conflito de interesses.

\section{Referências}

1. Brasil. Ministério da Saúde. Secretaria de Atenção à Saúde. Departamento de Atenção Básica. Saúde da criança: aleitamento materno e alimentação complementar. 2. ed. Brasília: Ministério da Saúde; 2015. 184 p.

2. World Health Organization. Expert consultation on the optimal duration of exclusive breastfeeding. In: World Health Organization. Global strategy for infant and young child feeding Geneva: WHO; 2001. p. 2. Disponível em: http://apps.who.int/gb/archive/pdf_files/WHA54/ea54id4.pdf 
3. Lamberti LM, Walker CLF, Noiman A, Victora C, Black RB. Breastfeeding and the risk for diarrhea morbidity and mortality. Bio Med Central Public Health 2011; 11(3):15.

4. Boccolini CS, Carvalho ML, Oliveira MIC, Boccolini PMM. O papel do aleitamento materno na redução das hospitalizações por pneumonia em crianças brasileiras menores de 1 ano. J Pediatr. 2011; 87(5):399-404.

5. Who collaborative study team on the role of breastfeeding on the prevention of infant mortality. Effect of breastfeeding on infant and child mortality due to infectious diseases in less developed countries: a pooled analysis. The Lancet 2000; 355(9202):451-455.

6. Victora CG, Barros AJD, França GVA, Bahl R, Rollins NC, Horton S, et al. Breastfeeding in the 21st century: epidemiology, mechanisms, and lifelong effect. Lancet 2016; 387(10017):475-90.

7. Rollins NC, Lutter CK, Bhandari N, Hajeebhoy N, Horton S, Martines JC, et al. Why invest, and what it will take to improve breastfeeding practices? Lancet 2016; 387(10017):491-504.

8. Collaborative group hormonal factors in breast cancer. Breast cancer and breastfeeding: collaborative reanalysis of individual data from 47 epidemiological studies in 30 countries, including 50.302 women with breast cancer and 96.973 women without the disease. The Lancet 2002; 360:187-95.

9. Danforth KN, Tworoger SS, Hecht JL, Rosner BA, Colditz GA, Hankinson SE. Breastfeeding and risk of ovarian cancer in two prospective cohorts. Cancer Causes and Control 2007; 18(5):517-523.

10. Kramer MS, Kakuma R. The optimal duration of exclusive breastfeeding: a systematic review. Geneva: WHO; 2001. 47 p.

11. Brasil. Ministério da Saúde. Pesquisa Nacional de Demografia e Saúde da Criança e da Mulher PNDS 2006: dimensões do processo reprodutivo e da saúde da criança. Brasília: Ministério da Saúde; 2009. 300 p.

12. Brasil. Ministério da Saúde. Secretaria de Atenção à Saúde. Departamento de Ações Programáticas e Estratégicas. II Pesquisa de Prevalência de Aleitamento Materno nas capitais brasileiras e Distrito Federal. Brasília: Ministério da Saúde; 2009. 108 p.

13. Venancio SI, Escuder MML, Kitoko P, Rea MF, Monteiro CA. Frequência e determinantes do aleitamento materno em municípios do Estado de São Paulo. Rev Saúde Pública 2002; 36(3):313-318.

14. França GVA, Brunken GS, Silva SM, Escuder MM, Venancio SI. Determinantes da amamentação no primeiro ano de vida em Cuiabá, Mato Grosso. Rev Saúde Pública 2007; 41(5):711-718.

15. Parada CMGL, Carvalhaes MABL, Jamas MT. Práticas de alimentação complementar em crianças no primeiro ano de vida. Rev Latino-Am Enfermagem 2007; 15(2):282-9.

16. Saldiva SRDM, Escuder MM, Mondini L, Levy RB, Venancio SI. Práticas alimentares de crianças de 6 a 12 meses e fatores maternos associados. J Pediatr. 2007; 83(1):53-58.

17. Caetano MC, Ortiz TT, Silva SGL, Souza FIS, Sarni ROS. Alimentação complementar: práticas inadequadas em lactentes. J Pediatr. 2010; 86(3):196-201. 
18. Gomes PTT. Práticas alimentares de crianças menores de um ano que compareceram na segunda etapa da campanha nacional de vacinação nos postos de saúde fixos da cidade de Guarapuava-PR, em 2004 [dissertação]. [Ribeirão Preto]: Escola de Enfermagem de Ribeirão Preto, Universidade de São Paulo; 2005.

19. Saldan PC, Venancio SI, Saldiva SRDM, Pina JC, Mello DF. Práticas de aleitamento materno de crianças menores de dois anos de idade com base nos indicadores da Organização Mundial da Saúde. Rev Nutr. 2015; 28(4):409-420.

20. Brecailo MK, Corso ACT, Almeida CCBA, Schmitz BAS. Fatores associados ao aleitamento materno exclusivo em Guarapuava, Paraná. Rev Nutr. 2010; 23(4):553-563.

21. Lwanga SK, Lemeshow S. Sample size determination in health studies: a practical manual. Geneva: WHO; 1991. 30 p.

22. Silva NN. Amostragem probabilística: um curso introdutório. São Paulo: EDUSP; 1998. 124 p.

23. Saulistiano LPQ, Diniz ALD, Abdallah VOS, Pinto RMC. Fatores associados à duração do aleitamento materno em crianças menores de seis meses. Rev Bras Ginecol Obstet. 2012; 34(1):28-33.

24. Pellegrinelli ALR, Pereira SCL, Ribeiro IP, Santos LC. Influência do uso de chupeta e mamadeira no aleitamento materno exclusivo entre mães atendidas em um Banco de Leite Humano. Rev Nutr. 2015; 28(6):631-639.

25. Tamasia GA, Venâncio SI, Saldiva SRDM. Situation of breastfeeding and complementary feeding in a medium-sized municipality in the Ribeira Valley, São Paulo. Rev Nutr. 2015; 28(2):143-153.

26. Campagnolo PDB, Louzada MLC, Silveira EL, Vitolo MR. Práticas alimentares no primeiro ano de vida e fatores associados em amostra representativa da cidade de Porto Alegre, Rio Grande do Sul. Rev Nutr. 2012; 25(4):431-439.

27. Ducci AL, Vannuchi MTO, Tacla MTGM, Souza SNDH, Reis TB. Prevalência e fatores associados ao aleitamento materno exclusivo em menores de seis meses no município de Rolândia - PR. Rev. Min. Enferm. 2013; 17(2):381-389.

28. Mauch CE, Scott JA, Magarey AM, Daniels LA. Predictors of and reasons for pacifier use in firsttime mothers: an observational study. BMC Pediatr. 2012; 12(7):1-10.

29. Buccini GS, Benício MH, Venancio SI. Determinantes do uso de chupeta e mamadeira. Rev Saúde Pública 2014; 48(4):571-582.

30. Dadalto ECV, Rosa EM. Fatores associados ao uso de chupeta por lactentes nascidos pré-termo. Rev CEFAC 2016; 18(3):601-612.

31. Soares MEM, Giugliani ERJ, Braun ML, Salgado ACN, Oliveira AP, Aguiar, PR. Uso de chupeta e sua relação com o desmame precoce em população de crianças nascidas em Hospital Amigo da Criança. J Pediatr. 2003; 79(4):309-16.

32. Saldiva SRDM, Venancio RI, Gouveia AGC, Castro ALS, Escuder MML, Giugliani ERJ. Influência regional no consumo precoce de alimentos diferentes do leite materno em menores de seis meses residentes nas capitais brasileiras e Distrito Federal. Cad Saúde Pública 2011; 27(11):2253-2262. 
33. Neves ACM, Moura EC, Santos W, Carvalho KMB.Factors associated with exclusive breastfeeding in the Legal Amazon and Northeast regions, Brazil, 2010. Rev Nutr. 2014; 27(1):81-95.

34. Chaves RG, Lamounier JA, César CC. Fatores associados com a duração do aleitamento materno. J Pediatr. 2007; 83(3):241-246.

35. Ramos CV, Almeida JAG, Alberto NSMC, Teles JBM, Saldiva SRDM. Diagnóstico da situação do aleitamento materno no Estado do Piauí, Brasil. Cad Saúde Pública 2008; 24(8):1753-1762.

36. Vieira GO, Almeida JAG, Silva LR, Cabral VA, Santana Netto PV. Fatores associados ao aleitamento materno e desmame em Feira de Santana, Bahia. Rev. Bras. Saúde Matern Infant. 2004; 4(2):143-150.

37. Boccolini CS, Carvalho ML, Oliveira MIC, Pérez-Escamilla R. Amamentação na primeira hora de vida e mortalidade neonatal. J Pediatr. 2013; 89(2):131-136.

38. Will TK, Arndt JG, Torres GG, Andrade JR, Pereira TSS, Molina MCB. Fatores de proteção para a amamentação na primeira hora de vida. Rev Bras Promoc Saúde 2013; 26(2):274-280.

39. Boccolini CS, Carvalho ML, Oliveira MIC, Vasconcellos AGG. Fatores associados à amamentação na primeira hora de vida. Rev Saude Publica 2011; 45(1):69-78.

40. Rea MF. Reflexões sobre a amamentação no Brasil: de como passamos a 10 meses de duração. Cad Saúde Pública 2003; 19(1):37-45.

Recebido: $16 / 12 / 2016$

Aceito: 20/2/2017 